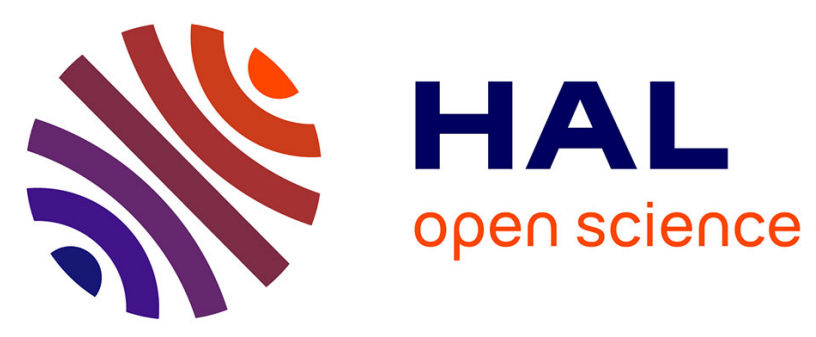

\title{
Motional heterogeneity in human acetylcholinesterase revealed by a non-Gaussian model for elastic incoherent neutron scattering Motional heterogeneity in human acetylcholinesterase revealed by a non-Gaussian model for elastic incoherent neutron scattering
} Judith Peters, Gerald R Kneller

\section{To cite this version:}

Judith Peters, Gerald R Kneller. Motional heterogeneity in human acetylcholinesterase revealed by a non-Gaussian model for elastic incoherent neutron scattering Motional heterogeneity in human acetylcholinesterase revealed by a non-Gaussian model for elastic incoherent neutron scattering. Journal of Chemical Physics, 2013, 139 (16), pp.165102. 10.1063/1.4825199 . hal-01582670

\author{
HAL Id: hal-01582670 \\ https://hal.science/hal-01582670
}

Submitted on 6 Sep 2017

HAL is a multi-disciplinary open access archive for the deposit and dissemination of scientific research documents, whether they are published or not. The documents may come from teaching and research institutions in France or abroad, or from public or private research centers.
L'archive ouverte pluridisciplinaire HAL, est destinée au dépôt et à la diffusion de documents scientifiques de niveau recherche, publiés ou non, émanant des établissements d'enseignement et de recherche français ou étrangers, des laboratoires publics ou privés. 


\section{AD $\mid \begin{aligned} & \text { The Journal of } \\ & \text { Chemical Physics }\end{aligned}$}

Motional heterogeneity in human acetylcholinesterase revealed by a non-Gaussian model for elastic incoherent neutron scattering

Judith Peters and Gerald R. Kneller

Citation: The Journal of Chemical Physics 139, 165102 (2013); doi: 10.1063/1.4825199

View online: http://dx.doi.org/10.1063/1.4825199

View Table of Contents: http://scitation.aip.org/content/aip/journal/jcp/139/16?ver=pdfcov

Published by the AIP Publishing

Advertisement:

AlP Re-register for Table of Content Alerts 


\title{
Motional heterogeneity in human acetylcholinesterase revealed by a non-Gaussian model for elastic incoherent neutron scattering
}

\author{
Judith Peters ${ }^{1}$ and Gerald R. Kneller ${ }^{2, \text { a) }}$ \\ ${ }^{1}$ Université Joseph Fourier, UFR PhITEM, BP 53, 38041 Grenoble, France; Institut Laue Langevin, BP 156, \\ 38042 Grenoble, France; and Institut de Biologie Structurale, 41 rue Jules Horowitz, 38027 Grenoble, France \\ ${ }^{2}$ Centre de Biophys. Moléculaire, CNRS, Rue Charles Sadron, 45071 Orléans, France; Synchrotron Soleil, \\ L'Orme de Merisiers, 91192 Gif-sur-Yvette, France; and Université d'Orléans, Chateau de la Source-Av. du \\ Parc Floral, 45067 Orléans, France
}

(Received 20 August 2013; accepted 1 October 2013; published online 22 October 2013)

\begin{abstract}
We study the dynamical transition of human acetylcholinesterase by analyzing elastic neutron scattering data with a simulation gauged analytical model that goes beyond the standard Gaussian approximation for the elastic incoherent structure factor [G. R. Kneller and K. Hinsen, J. Chem. Phys. 131, 045104 (2009)]. The model exploits the whole available momentum transfer range in the experimental data and yields not only a neutron-weighted average of the atomic mean square position fluctuations, but also an estimation for their distribution. Applied to the neutron scattering data from human acetylcholinesterase, it reveals a strong increase of the motional heterogeneity at the two transition temperatures $T=150 \mathrm{~K}$ and $T=220 \mathrm{~K}$, respectively, which can be located with less ambiguity than with the Gaussian model. We find that the first transition is essentially characterized by a change in the form of the elastic scattering profile and the second by a homogeneous increase of all motional amplitudes. These results are in agreement with previous combined experimental and simulation studies of protein dynamics, which attribute the first transition to an onset of methyl rotations and the second to more unspecific diffusion processes involving large amplitude motions. (C) 2013 AIP Publishing LLC. [http://dx.doi.org/10.1063/1.4825199]
\end{abstract}

\section{INTRODUCTION}

The relation between function and dynamical properties of proteins is one of the most important and fascinating research topics in molecular biophysics and biochemistry. First valuable insights into protein dynamics could be obtained from Mößbauer and neutron scattering studies of myoglobin. ${ }^{1,2}$ Mößbauer spectroscopy is sensitive to the motion of particular atoms, in case of myoglobin the iron atom bound to the heme group, whereas neutron scattering gives an averaged view over the single-particle dynamics of all hydrogen atoms in the protein under consideration. Both methods reveal a marked increase of the mean-square atomic position fluctuations (MSPFs) at a temperature of about $220 \mathrm{~K}$, the socalled transition temperature, as well as an onset of diffusive motions. Referring to Frauenfelder's picture of a "rugged" multi-minima (free) energy landscape, ${ }^{3}$ the dynamical transition has been explained by the picture of thermal activation out of these minima (conformational substates) in which they are trapped at low temperatures. Most elastic neutron scattering studies of protein dynamics are analyzed by using the concept of a "representative atom" moving isotropically in a harmonic potential around its equilibrium position. This assumption leads to a simple Gaussian model for the elastic incoherent structure factor (EISF) as a function of the momentum transfer (see Refs. 4 and 5 and references herein). Using the picture of an effective quadratic (free) energy surface, the dynamical transition can be qualitatively explained

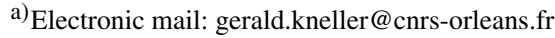

by a curvature change of this surface. The assumption that a single atom can represent the dynamics of all hydrogen atoms in a given protein is though not realistic. Hydrogen atoms are homogeneously distributed over the whole protein, including methyl groups and side chains in general, and the atomic dynamics probed in neutron scattering experiments is thus characterized by an important motional heterogeneity. Molecular dynamics (MD) simulation is a useful tool to investigate the impact of these effects and others in "virtual" scattering experiments. ${ }^{6-11}$ In Ref. 9 it has been in particular shown that a form analysis of simulated EISFs with a simple model leads to a realistic form for the distribution function of the atomic position fluctuations and that all moments are represented by simple analytical expressions in the fit parameters.

The idea of this paper is to use the model proposed in Ref. 9 on experimental elastic neutron scattering data obtained from human acetylcholinesterase (hAChE) on three instruments of the Institut Laue-Langevin (ILL) in Grenoble. Cholinesterases are key enzymes in the nervous systems in mammalians that catalyze the hydrolysis of the neurotransmitter acetylcholine, in order to terminate neurotransmission at cholinergic synapses and to reset an excited neuron back to its resting state after activation. Elastic neutron scattering data on different cholinesterases have been recently analyzed with the widely used Gaussian model. ${ }^{12-14}$

\section{THEORY}

The experimental data used in the present study have been obtained from $\mathrm{D}_{2} \mathrm{O}$-hydrated protein powders. ${ }^{12}$ Since 
the cross section for incoherent scattering from hydrogen atoms is dominant and since hydrogen atoms make up about $50 \%$ of the total number of atoms in a protein, the measured dynamic structure factor can be approximated as

$$
S(\boldsymbol{q}, \omega) \approx \frac{1}{2 \pi} \int_{-\infty}^{+\infty} d t \exp (-i \omega t) F_{H}(\boldsymbol{q}, t),
$$

where $F_{H}(\boldsymbol{q}, t)$ is the incoherent intermediate scattering function of the hydrogen atoms:

$$
F_{H}(\boldsymbol{q}, t)=\frac{1}{N_{H}} \sum_{\alpha \in H}\left\langle\exp \left(-i \boldsymbol{q} \cdot \boldsymbol{R}_{\alpha}(0)\right) \exp \left(i \boldsymbol{q} \cdot \boldsymbol{R}_{\alpha}(t)\right)\right\rangle \text {. }
$$

The variables $\boldsymbol{q}$ and $\omega$ denote, respectively, the momentum and energy transfer from the neutron to the sample in units of $\hbar$, and $\boldsymbol{R}_{\alpha}$ is the position operator of hydrogen atom $\alpha$. The atomic motions in hydrated protein powders are confined in space since the diffusion of whole proteins is suppressed in such systems. In this case the intermediate scattering function tends to a plateau value for long times,

$$
\lim _{t \rightarrow \infty} F_{H}(\boldsymbol{q}, t)=\frac{1}{N_{H}} \sum_{\alpha \in H} \mid\left\langle\left.\exp \left(i \boldsymbol{q} \cdot \boldsymbol{R}_{\alpha}\right\rangle\right|^{2} \equiv \operatorname{EISF}(\boldsymbol{q}),\right.
$$

which is the elastic incoherent structure factor determining the elastic component of the dynamic structure factor:

$$
S_{e l}(\boldsymbol{q}, \omega)=\operatorname{EISF}(\boldsymbol{q}) \delta(\omega) .
$$

To model elastic incoherent neutron scattering from hydrated protein powders we make the first assumption that the motion of any (hydrogen) atom in the system can be described by diffusive motion in an isotropic harmonic potential. In this case the corresponding EISF has the simple Gaussian form

$$
E I S F_{\alpha}(\boldsymbol{q})=\exp \left(-q^{2}\left\langle u_{q, \alpha}^{2}\right\rangle\right),
$$

where $q \equiv|\boldsymbol{q}|$ and $u_{q, \alpha}$ is the projection of its displacement with respect to the average position onto the momentum transfer vector $\boldsymbol{q}$ :

$$
u_{\alpha, q}=\boldsymbol{n}_{q} \cdot\left(\boldsymbol{R}_{\alpha}-\left\langle\boldsymbol{R}_{\alpha}\right\rangle\right) .
$$

Here, $\boldsymbol{n}_{q}$ is the unit vector in the direction of $\boldsymbol{q}$. Since the potential is isotropic, the fluctuation of $u_{\alpha, q}$ does not depend on the direction of projection, such that

$$
\left\langle u_{\alpha, q}^{2}\right\rangle=\frac{\left\langle u_{\alpha, x}^{2}\right\rangle+\left\langle u_{\alpha, y}^{2}\right\rangle+\left\langle u_{\alpha, x}^{2}\right\rangle}{3} .
$$

For small values of $\boldsymbol{q}$,

$$
q^{2}\left\langle u_{\alpha, q}^{2}\right\rangle \ll 1
$$

the isotropic Gaussian approximation always holds. ${ }^{11}$

The second assumption is that the motional heterogeneity can be described by a Gamma distribution for the individual MSPFs. Defining the dimensionless momentum transfer

$$
\tilde{q}=\sigma q,
$$

where $\sigma>0$ is a scale variable with the dimension of a length, we follow Ref. 9 and express the total EISF (the index $m$ stands for "model") in the form

$$
E I S F_{m}(\tilde{q} ; \beta)=\int_{0}^{\infty} d \lambda p(\lambda ; \beta) \exp \left(-\lambda \tilde{q}^{2}\right),
$$

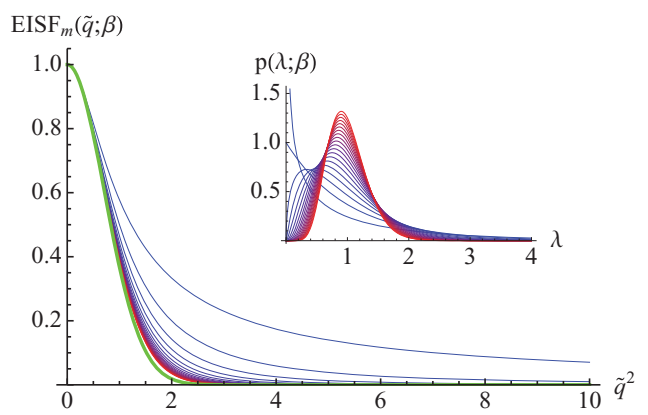

FIG. 1. Function $E I S F_{m}(\tilde{q} ; \beta)$ and corresponding distribution $p(\lambda ; \beta)$ (inset) for $\beta=n / 2$, setting $n=1, \ldots, 20$ (blue to red). The thick green line corresponds to the Gaussian limit $\operatorname{EISF}_{G A}(q)=\exp \left(-\tilde{q}^{2}\right)$. The inset shows the corresponding distributions $p(\lambda, \beta)$, where $p(\lambda, \infty)=\delta(\lambda-1)$ corresponding to the Gaussian limit is not shown.

where $p(\lambda ; \beta)$ is the Gamma distribution,

$$
p(\lambda ; \beta)=\frac{\beta \exp (-\beta \lambda)(\beta \lambda)^{\beta-1}}{\Gamma(\beta)} \quad(0<\beta<\infty),
$$

and $\lambda$ is dimensionless (see Fig. 1). Here, $\Gamma(\beta)$ denotes the Gamma function. ${ }^{15}$ Setting

$$
\lambda=\frac{u^{2}}{\sigma^{2}} \equiv \tilde{u}^{2}
$$

one recognizes that $p(\lambda ; \beta)$ can be interpreted as a distribution for physical MSPFs. The integral (10) can be performed analytically and yields a simple analytical form for the model EISF:

$$
\operatorname{EISF}_{m}(\tilde{q} ; \beta)=\frac{1}{\left(1+\frac{\tilde{q}^{2}}{\beta}\right)^{\beta}} .
$$

The standard Gaussian form is retrieved in the limit $\beta \rightarrow \infty$,

$$
E I S F_{m}(\tilde{q} ; \beta) \stackrel{\beta \rightarrow \infty}{\rightarrow} \exp \left(-\tilde{q}^{2}\right)
$$

and corresponds to perfect homogeneity in the atomic motions:

$$
\lim _{\beta \rightarrow \infty} p(\lambda ; \beta)=\delta(\lambda-1) .
$$

According to Eqs. (10) and (12) the model EISF is the generating function for the moments and cumulants of $\lambda=\tilde{u}^{2}$ :

$$
\tilde{c}_{k}=\left.(-1)^{k} \frac{\partial^{k} \ln \left(E I S F_{m}(\tilde{q} ; \beta)\right)}{\partial\left(\tilde{q}^{2}\right)^{k}}\right|_{q=0}=\frac{(k-1) !}{\beta^{k-1}} .
$$

Noting that the physical atomic displacements are given by $u=\sigma \tilde{u}$, if follows that $c_{k}=\sigma^{2 k} \tilde{c}_{k}$, and for $k=1,2$ one obtains in particular,

$$
\begin{gathered}
c_{1}=\left\langle u^{2}\right\rangle=\sigma^{2}, \\
c_{2}=\left\langle\left(u^{2}-\left\langle u^{2}\right\rangle\right)^{2}\right\rangle=\frac{\sigma^{4}}{\beta} .
\end{gathered}
$$

Here, $\left\langle u^{2}\right\rangle$ is to be understood in the sense of Eq. (7) and the standard deviation of $\left\langle u^{2}\right\rangle$ is given by the square root of $c_{2}$ :

$$
\Sigma\left(\left\langle u^{2}\right\rangle\right)=\frac{\sigma^{2}}{\sqrt{\beta}} .
$$


TABLE I. Characteristics of the neutron scattering spectrometers used in this study.

\begin{tabular}{lccc}
\hline \hline & IN6 & IN13 & IN16 \\
\hline$\Delta E(\mu \mathrm{eV})$ & 50 & 8 & 0.9 \\
$q$-range $(1 / \AA)$ & $0.38-1.6$ & $0.19-4.9$ & $0.43-1.93$ \\
\hline \hline
\end{tabular}

We note here that expression (13) is referred to as "Model B" in Ref. 9. In "Model A" the Gamma distribution (11) is, in fact, replaced by a shifted version, where the shift accounts for a minimum value for the MSPFs. We found, however, that this parameter cannot be determined from experimental data with sufficient statistical accuracy.

\section{RESULTS}

The experimental data on $\mathrm{D}_{2} \mathrm{O}$-hydrated hAChE powder samples on which the present study is based have been collected recently on the spectrometers IN6, IN13, and IN16 of the ILL. ${ }^{13}$ We focus here on an analysis of these data with the model described in Sec. II. The resolution and the accessible $q$-range of the three instruments are resumed in Table I. Figures 2 and 3 display experimental EISFs from hAChE which have been obtained from the IN13 spectrometer, together with the fits of model (13) and its Gaussian approximation (14), respectively. Both models are fitted for the same selection of five different temperatures. In these fits we included an additional amplitude factor to account for the fact that $\operatorname{EISF}(0)<1$ due to multiple scattering and absorption effects. $^{16,17}$ The vertical dashed line in Fig. 3 delimits the region

$$
0 \leq q \leq 1.3 \AA^{-1},
$$

which has been used for the fit of the Gaussian approximation. The choice of the fit domain (20) is to some extent arbitrary and has been chosen as a compromise between condition (8) and the necessity to include a minimal number of data points in the fit. The figure demonstrates that only a small fraction of the available EISF data can be used with the Gaussian approximation.

Fig. 4 shows the MSPFs which have been obtained from the fits of model (13) to the elastic scattering intensities used in this study. In order to reduce the statistical noise, the values have been averaged over two neighboring data points.

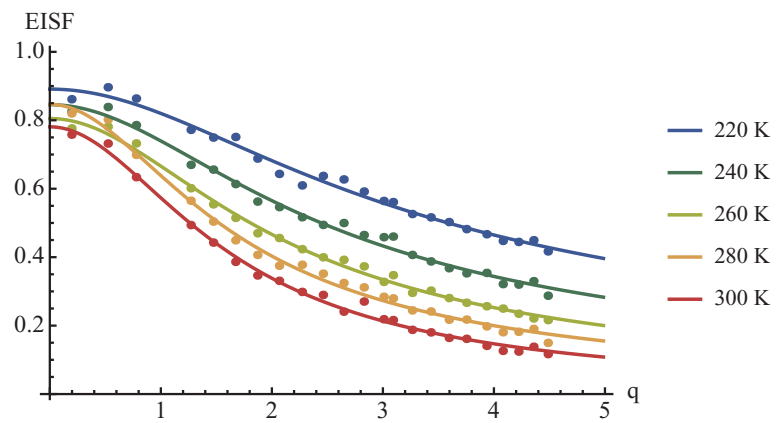

FIG. 2. Elastic incoherent structure factor of hydrated hAChE powders obtained by the IN13 spectrometer at the ILL in Grenoble (dots) and fits of expression (13).

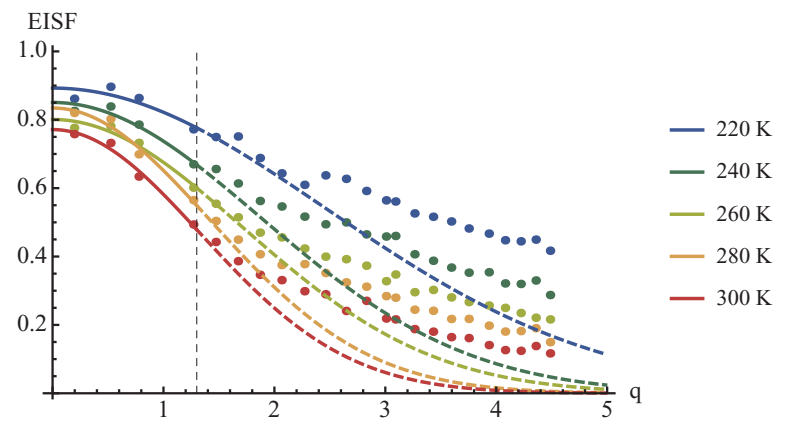

FIG. 3. Same as Fig. 2, but with fits of the Gaussian approximation (14) for the elastic intensity. The vertical dashed line indicates the upper limit of the fit region.

It is well visible that the extracted MSPFs become smaller with decreasing energy resolution (see Table I). The reason is that spurious contributions from quasi-elastic scattering contribute increasingly to the elastic line, such that the contributions from real elastic scattering seemingly decrease. The effect is well known and several correction methods implying the Gaussian approximation of the EISF have been proposed in the recent literature. ${ }^{7,18,19}$ Using model (13), we find the protein transition temperature of hAChE on all instruments at the same temperature, $T=220 \mathrm{~K}$, despite the fact that the extracted motional amplitudes are different. The inset of Fig. 4 displays a zoom on the MSPFs obtained from IN13. It exhibits also a first dynamical transition at about $T=150 \mathrm{~K}$, which has been explained in earlier work by the onset of methyl rotations. ${ }^{20}$ We attribute this detail to the large momentum transfer range of the IN13 spectrometer, which is particularly suitable to study methyl rotations. At higher temperatures the MSPFs obtained from IN16 show a remarkable drop, which has also been observed in studies of highly concentrated protein solutions close to the conditions of denaturation. ${ }^{21}$ The available space for atomic motion thus effectively decreases, although the temperature increases. For a quasi-harmonic system one would expect $\left\langle u^{2}\right\rangle \propto T$, and the observed drop of the MSPFs with increasing temperature thus clearly indicates a non-harmonic regime. Admitting an arbitrary form of $\sigma(T)$, this is though still compatible with the Gaussian form for the EISF of the individual atoms which is assumed in our model.

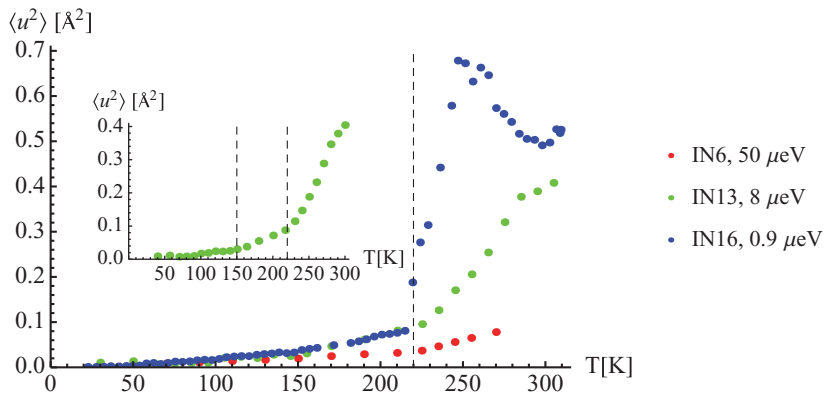

FIG. 4. MSPFs for hydrated hAChE powders obtained from different spectrometers at the ILL in Grenoble by fits of model (13). The vertical dashed lines are guides to the eye indicating the dynamical transitions at $150 \mathrm{~K}$ and $220 \mathrm{~K}$ and the inset displays the data obtained from IN13 separately. 


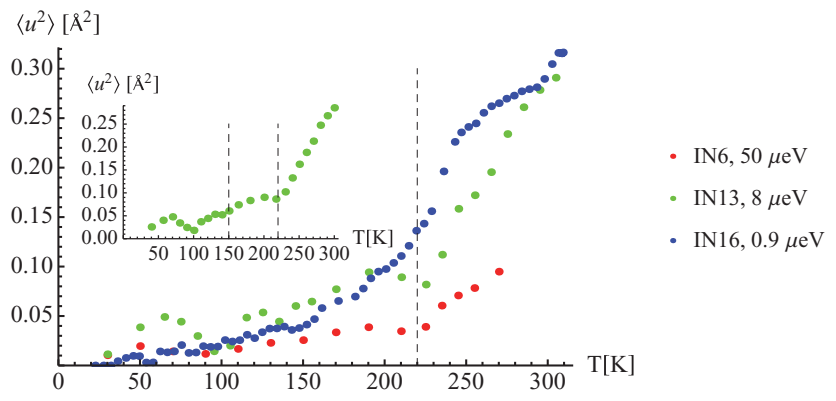

FIG. 5. Same as Fig. 4, but with fits of the Gaussian model (14) for the elastic intensity. All fits have been performed with the $q$-range used in Fig. 3.

It is worthwhile noting that the MSPFs obtained from IN13 do not exhibit the drop mentioned above, but rather a drop in slope. The latter is, moreover, shifted to higher temperatures. No drop at all is obtained from the analysis of the IN6 data which cover the relevant temperature range, however, only partially. We attribute these differences to the decreasing instrumental energy resolution. As a result, the ratio of real elastic scattering to spurious contributions from quasi-elastic scattering is more and more reduced and the extracted MSPFs become correspondingly less and less sensitive to changes of the physical system parameters.

All the above features concerning temperature dependence of the MSPFs are less well discriminated by a data analysis with the Gaussian model, which is presented in Fig. 5. The fits have been performed for the $q$-range (20) and the same smoothing procedure as in Fig. 4 has been applied. Here the main transition at about $T=220 \mathrm{~K}$ does, for example, not clearly appear in the analysis of the IN16 data and the inset does not show the pre-transition at $150 \mathrm{~K}$. The extracted MSPFs are moreover considerably smaller than those in Fig. 4 and we have good reasons to believe that the latter values are closer to reality. In the MD simulation study, ${ }^{9}$ where model (13) has been used on simulated EISF data for lysozyme, the neutron-weighted MSPF is found to be $\left\langle u^{2}\right\rangle_{\mathrm{MD}} \approx 0.6 \AA^{2}$ at room temperature, whereas $\left\langle u^{2}\right\rangle_{\text {fit }} \approx 0.4 \AA^{2}$ is found for the corresponding fit with model (13) (referred to as "Model B" in Ref. 9). Since the fitted atom-averaged MSPF in this study is still underestimated by about $30 \%$, the values shown in Fig. 5 seem at least underestimated by a factor of 2 .

Fig. 6 presents the standard deviations corresponding to the atom-averaged MSPFs given in Fig. 4. The MSPFs have been constructed according to Eq. (19) and the results have

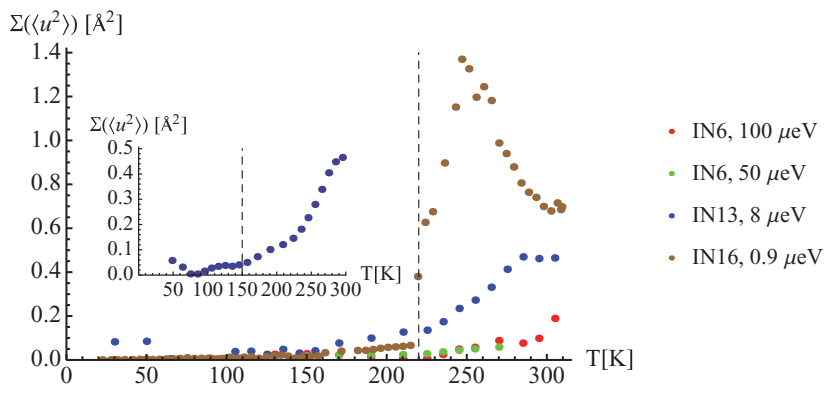

FIG. 6. Standard deviation of the MSPFs shown in Fig. 4. The inset shows again the IN13 data separately.
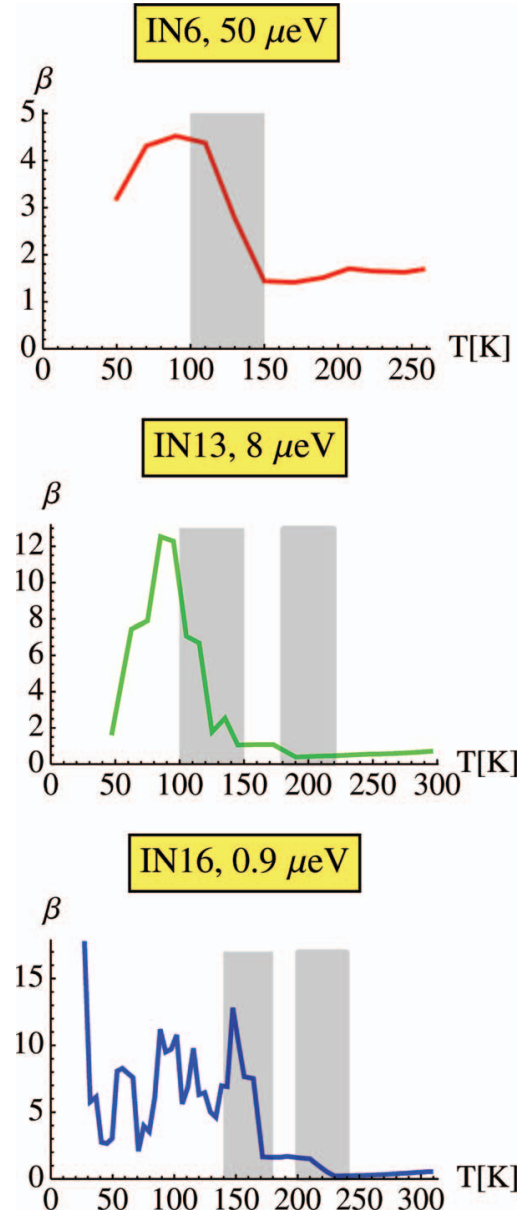

FIG. 7. $\beta$-parameters corresponding to the standard deviations shown in Fig. 6.

been smoothed with the same procedure as for Figs. 4 and 5 . The inset displays again the values for IN13. Knowing that $\Sigma\left(\left\langle u^{2}\right\rangle\right)=\left\langle u^{2}\right\rangle / \sqrt{\beta}$ within the proposed model, it is worthwhile tracing separately the $\beta$-parameter as a function of temperature. The results are displayed in Fig. 7. The displayed $\beta(T)$ curves have been smoothed using a moving average with a window of four data points. The shaded rectangles label regions of decay of the $\beta$-parameter, which signify a change in the form of the EISF. For all three instruments one observes such a region between roughly $100 \mathrm{~K}$ and $160 \mathrm{~K}$ and on IN13 and IN16 a second region between roughly 180 and $220 \mathrm{~K}$. Although the fit parameters exhibit fluctuations due to statistical errors in the data, energy resolution, and finite $q$-ranges, Figs. 6 and 7 clearly indicate an increase of motional heterogeneity around the two transition temperatures. It should be noted that increase at $T=150 \mathrm{~K}$ is accompanied by a strong drop in the $\beta$-parameter, corresponding thus to a strong change in the form of the EISF. This finding is perfectly coherent with the observation mentioned above that the transition at $150 \mathrm{~K}$ is due to the onset of methyl rotations with a broad distribution of rotational barriers. ${ }^{20}$ The main dynamical transition at $T=220 \mathrm{~K}$ is, in contrast, characterized by a smaller drop of the parameter $\beta$, which is only visible in the analysis of the IN13 and IN16 data. This transition is therefore essentially described by an increase of the scale parameter 
$\sigma$ due to a homogenous increase of all motional amplitudes. This result is in agreement with the observation made in a recent combined neutron and dielectric spectroscopy study, ${ }^{22}$ which indicates that there is, in fact, no important change in the characteristics of protein dynamics at the main transition temperature of about $220 \mathrm{~K}$, where less specific diffusion and relaxation processes involving large amplitude motions come into play. We mention here side chain motions, which could be responsible for the slight increase of the motional heterogeneity at $T=220 \mathrm{~K}$, since atoms at the end of the side chain exhibit often larger motional amplitudes than those close to the backbone. ${ }^{23,24}$

\section{DISCUSSION AND CONCLUSION}

We have tested a simulation-based model for elastic neutron scattering intensities on experimental data from human acetylcholinesterase. The data had been previously recorded on different spectrometers of the Institut Laue-Langevin in Grenoble. In contrast to the widely used Gaussian approximation for the EISF, the model used in this study permits to exploit the whole available $q$-range of the experimental EISF data. It accounts in particular for motional heterogeneity in the atomic mean-square position fluctuations, which is extracted by a form analysis of the EISF profile. Since neutron scattering is essentially probing the single particle dynamics of hydrogen atoms, a fraction of which is located in methyl groups and at the ends of mobile side chains, the extracted motional heterogeneity can be expected to be somewhat higher than for a hypothetic experiment in which all atoms are equally weighted. Contributions from deuterated hydration water should be small for powder samples.

The atom-averaged mean-square position fluctuations obtained from the proposed model clearly show two dynamical transitions at $T=150 \mathrm{~K}$ and $T=220 \mathrm{~K}$, respectively, which appear more clearly than in the analysis with the Gaussian model. Simulation studies indicate also that the resulting MSPFs are closer to the real values than those obtained from the Gaussian model. Our data analysis reveals in particular an increase of the motional heterogeneity at the two transition temperatures. The first transition at $T=150 \mathrm{~K}$ is essentially characterized by a drop of the $\beta$-parameter, indicating a change in the form of the EISF. The second transition at $T=220 \mathrm{~K}$ is, in contrast, essentially characterized by a change of the scale $\sigma$-parameter, indicating a more or less homogeneous increase of all atomic motions. These results are coherent with earlier combined experimental and simula- tion studies, which attribute the first transition to an onset of methyl rotations and the second to an onset of less specific diffusion processes involving large amplitude motions of the protein under consideration.

Although the proposed model for elastic scattering from proteins involves only one additional parameter compared to the Gaussian approximation, the gain in information is important. The proposed model seems sufficiently robust to be used for routine analyses of elastic neutron scattering spectra from hydrated protein powders and similar soft matter systems.

\section{ACKNOWLEDGMENTS}

J.P. would like to thank F. Nachon, M. Trovaslet, and P. Masson for the permission to use the experimental data for the presented study.

${ }^{1}$ H. Hartmann, F. Parak, W. Steigemann, G. Petsko, D. Ponzi, and H. Frauenfelder, Proc. Natl. Acad. Sci. U.S.A. 79, 4967 (1982).

${ }^{2}$ W. Doster, S. Cusack, and W. Petry, Nature (London) 337, 754 (1989).

${ }^{3}$ H. Frauenfelder, S. G. Sligar, and P. G. Wolynes, Science 254, 1598 (1991).

${ }^{4}$ G. Zaccai, Science 288, 1604 (2000).

${ }^{5}$ F. Gabel, D. Bicout, U. Lehnert, M. Tehei, M. Weik, and G. Zaccai, Q. Rev. Biophys. 35, 327 (2002).

${ }^{6}$ A. Tokuhisa, Y. Joti, H. Nakagawa, A. Kitao, and M. Kataoka, Phys. Rev. E 75, 041912 (2007).

${ }^{7}$ G. R. Kneller and V. Calandrini, J. Chem. Phys. 126, 125107 (2007).

${ }^{8}$ L. Meinhold, D. Clement, M. Tehei, R. Daniel, J. Finney, and J. Smith, Biophys. J. 94, 4812 (2008).

${ }^{9}$ G. R. Kneller and K. Hinsen, J. Chem. Phys. 131, 045104 (2009).

${ }^{10}$ Z. Yi, Y. Miao, J. Baudry, N. Jain, and J. C. Smith, J. Phys. Chem. B 116, 5028 (2012)

${ }^{11}$ G. R. Kneller and G. Chevrot, J. Chem. Phys. 137, 225101 (2012).

${ }^{12}$ J. Peters, M. Trovaslet, M. Trapp, F. Nachon, F. Hill, E. Royer, F. Gabel, L. van Eijck, P. Masson, and M. Tehei, Phys. Chem. Chem. Phys. 14, 6764 (2012).

${ }^{13}$ M. Trapp, M. Trovaslet, F. Nachon, M. M. Koza, L. van Eijck, F. Hill, M. Weik, P. Masson, M. Tehei, and J. Peters, J. Phys. Chem. B 116, 14744 (2012).

${ }^{14}$ M. Trovaslet, M. Trapp, M. Weik, F. Nachon, P. Masson, M. Tehei, and J. Peters, Chem. Biol. Interact. 203, 14 (2013).

${ }^{15}$ NIST Handbook of Mathematical Functions, edited by F. W. J. Olver, D. W. Lozier, R. F. Boisvert, and C. W. Clark (Cambridge University Press, 2010).

${ }^{16}$ R. Zorn, Nucl. Instrum. Methods Phys. Res. A 572, 874 (2007).

${ }^{17}$ R. Zorn, Nucl. Instrum. Methods Phys. Res. A 603, 439 (2009).

${ }^{18}$ T. Becker, J. A. Hayward, J. L. Finney, R. M. Daniel, and J. C. Smith, Biophys. J. 87, 1436 (2004).

${ }^{19}$ D. Vural and H. R. Glyde, Phys. Rev. E 86, 011926 (2012).

${ }^{20}$ J. Roh, V. Novikov, R. Gregory, J. Curtis, Z. Chowdhuri, and A. Sokolov, Phys. Rev. Lett. 95, 038101 (2005).

${ }^{21}$ M. Hennig, F. Roosen-Runge, F. Zhang, S. Zorn, M. W. A. Skoda, R. M. J. Jacobs, T. Seydel, and F. Schreiber, Soft Matter 8, 1628 (2012).

${ }^{22}$ S. Khodadadi, S. Pawlus, J. H. Roh, V. Garcia Sakai, E. Mamontov, and A. P. Sokolov, J. Chem. Phys. 128, 195106 (2008).

${ }^{23}$ S. Furois-Corbin, J. C. Smith, and G. R. Kneller, Proteins 16, 141 (1993).

${ }^{24}$ G. R. Kneller and J. C. Smith, J. Mol. Biol. 242, 181 (1994). 